\title{
Synthesis of 1,2,3,4-substituted spiroheterocyclic tetrahydroisoquinoline-1-ones and their structural similarity in water solution and in crystallohydrate solid state
}

\author{
Malinka P. Stoyanova, ${ }^{*}$ Silvia E. Angelova, ${ }^{a}$ Petar Y. Petrov, \\ Rosica P. Nikolova, ${ }^{\mathrm{C}}$ and Boris L. Shivachev ${ }^{\mathrm{c}}$ \\ ${ }^{a}$ Bulgarian Academy of Sciences, Institute of Organic Chemistry with Centre of Phytochemistry, \\ 1113 Sofia, Bulgaria \\ ${ }^{b}$ Sofia University, Faculty of Chemistry, 1164 Sofia, Bulgaria \\ ${ }^{c}$ Bulgarian Academy of Sciences, Central Laboratory of Mineralogy and Crystallography, \\ 1113 Sofia, Bulgaria \\ E-mail: mstoyanova@orgchm.bas.bg
}

\begin{abstract}
The title compounds were synthesized starting from homophthalic anhydride and a ketimine. The structure of 1'-methyl-1-oxo-2-(pyridine-3-yl-methyl)-2,4-dihydro-1H-spiro[isoquinoline-3,4'piperidine]-4-carboxylic acid was studied by X-ray analysis, ab initio and DFT calculations and the similarity between the conformation in crystalline hydrate state and in water solution was inferred. Derivatives of the parent compound were synthesized and studied by means of theoretical calculations.
\end{abstract}

Keywords: 3-Spiroheterocyclic tetrahydroisoquinolinone, homophthalic anhydride, ketimine, carboxamides, $a b$ initio and DFT calculations, crystal structure

\section{Introduction}

Tetrahydroisoquinolines are a wide class of compounds with a broad range of biological activities including antitumor, ${ }^{1}$ antibacterial, antiallergic, and psychotropic. ${ }^{2}$ The tetrahydroisoquinoline fragment is present in a number of plant alkaloids. ${ }^{3}$ In addition these compounds are also attractive objects for stereochemical studies, since they display variable conformational behavior depending on the substituents that are introduced. ${ }^{4}$ Their synthesis is a field of ongoing interest for synthetic organic chemistry because of their properties and for elucidation of the structure-activity relationship. ${ }^{5}$ Various methods for the synthesis of tertrahydroisoquinolines are known; amongst them is the reaction of homophthalic anhydride with imines. ${ }^{6}$ This method has the advantage of constructing the tetrahydroisoquinoline fragment and introducing three substituents in a single step. 
As continuation of our studies of new heterocyclic compounds with expected pharmacological activities, ${ }^{4,6 a, b, 7}$ emphasizing the scope and limitations of the reaction between homophthalic anhydride and compounds containing activated double bonds, a series of new tetrahydroisoquinolinones was synthesized. In a recent paper ${ }^{4}$ we described the conformations of 3-(furan-3-yl)-2-phenethyl-4-(pyrrolidine-1-carbonyl)-3,4-dihydroisoquinolin-1(2H)-one deduced by X-ray analysis, dynamic NMR techniques and $a b$ initio and DFT calculations.

Here we present the synthesis of 1'-methyl-1-oxo-2-(pyridine-3-yl-methyl)-2,4-dihydro- $1 \mathrm{H}$ spiro[isoquinoline-3,4'-piperidine]-4-carboxylic acid $\mathbf{1}$ and its derivatives. The structures of the obtained compounds were studied by X-ray analysis, $a b$ initio and DFT calculations. So far in the literature, few 3-spiroisoquinoline carboxylic acids synthesized by reaction of homophthalic anhydride and ketimines ${ }^{6 c, 8}$ are described but none of them are prepared from an imine originating from heterocyclic ketones.

\section{Results and Discussion}

The acid 1 was synthesized by reaction of homophthalic anhydride 2 and the heterocyclic ketimine 3 ( $N$-(methylpiperidine-4-ylidene)- $N$-(pyridin-3-ylmethyl)amine) (Scheme 1) and was isolated in analytically pure form by filtration and simple washing with $\mathrm{CH}_{2} \mathrm{Cl}_{2}$ (93\% yield). Upon standing in air the compound gained one and a half molecules of water and changed its composition to (acid) $2_{2}\left(\mathrm{H}_{2} \mathrm{O}\right)_{3}$. As expected and indicated by IR spectra (two bands at 1410 and $1610 \mathrm{~cm}^{-1}$ assigned for the symmetrical and antisymmetrical vibration of carboxylate anion) and $\mathrm{X}$-ray analysis (see Figure 1) the acid $\mathbf{1}$ exist as a zwitterion.

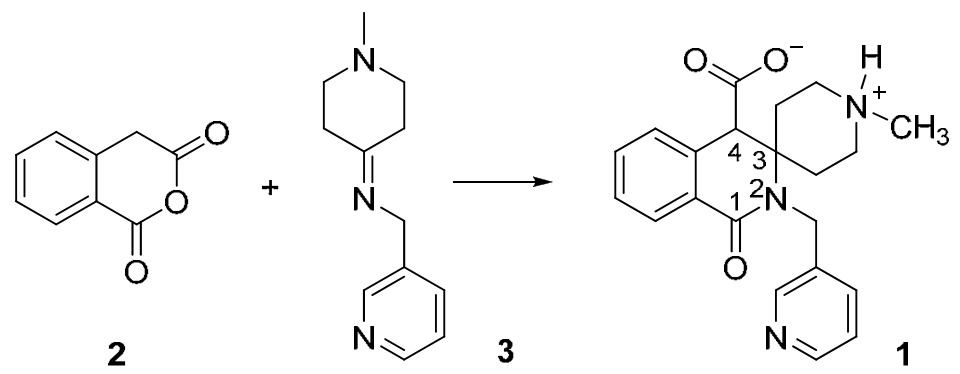

Scheme 1. Synthesis of carboxylic acid 1: $\mathrm{ClCH}_{2} \mathrm{CH}_{2} \mathrm{Cl}$, rt, $5 \mathrm{~h}$.

The structure of $\mathbf{1}$ in solid state was characterized by single-crystal X-ray diffraction analysis $^{9}$ (Figure 1). Single crystals of $\mathbf{1}$ with four water molecules in an asymmetric unit were obtained by slow evaporation from water/methanol solution. The crystals were isolated directly from the solution and mounted on a capillary. If kept in air outside the solution, spontaneous loss of water occurs, the crystals degrade and the composition reverts to $(\operatorname{acid})_{2}\left(\mathrm{H}_{2} \mathrm{O}\right)_{3}$. The bond lengths and angles of $\mathbf{1}$ are comparable to those observed in other quinoline derivatives. ${ }^{10}$ The charge transfer effect is expressed through the similarity of the C-O distances of 1.243(4) and 
1.252(4) A within the carboxyl group. The tetrahydroisoquinolin-1-one and picolyl ring systems are nearly planar with respective rms deviation of 0.164(5) and 0.007(5) $\AA$. They are attached to each other in a conformationally fluxional way; the dihedral angle between their mean planes is $83.74(8)^{\circ}$. The piperidine adopts a chair conformation, moving the spiro-carbon atom out of the quinolinone plane by $0.355(2) \AA$. The tetrahydroisoquinoline-1-one moieties build up chains parallel to $b$ through a single N3-H3N...O3 charge assisted hydrogen bond (Table 1). In the asymmetric unit of $\mathbf{1}$ each molecule is surrounded by four water molecules.

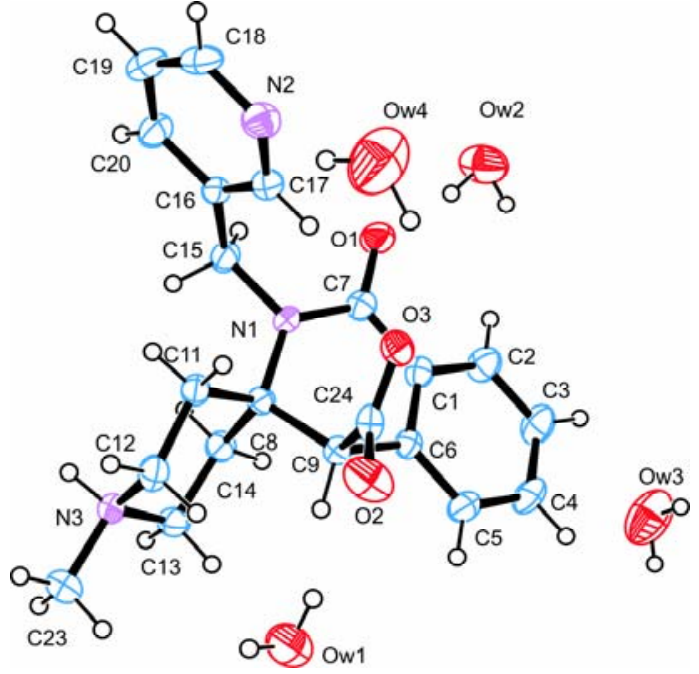

A

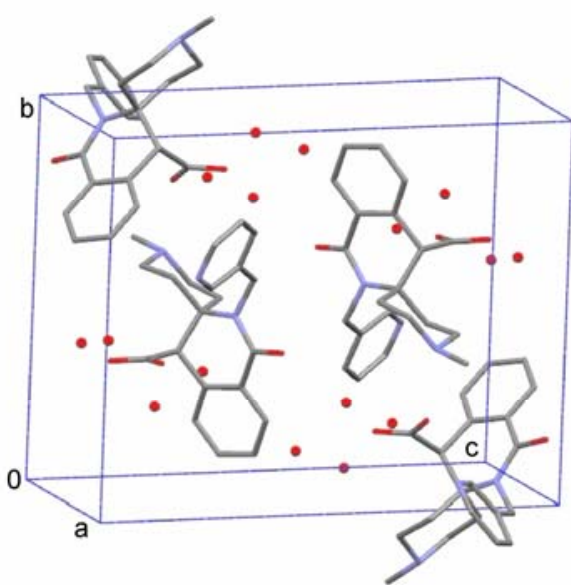

B

Figure 1. (A) ORTEP drawing of the basic crystallographic unit of acid 1 (S-enantiomer is shown), the hydrogen atoms are shown as small sphere of arbitrary radii. (B) A view of the molecular packing in $1 \times 4 \mathrm{H}_{2} \mathrm{O}$. All hydrogen atoms have been omitted for clarity.

Table 1. Hydrogen bonds $(\AA)$ in 1

\begin{tabular}{|c|c|c|c|c|}
\hline$D-\mathrm{H} \cdots A$ & $D-\mathrm{H}$ & $H \cdots A$ & $D \cdots A$ & $D-\mathrm{H}^{\cdots} \cdots A\left({ }^{\circ}\right)$ \\
\hline $\mathrm{N} 3-\mathrm{H} 3 \mathrm{~N} \cdots \mathrm{O} 3^{\mathrm{i}}$ & 0.95 & 1.901 & $2.706(4)$ & 141.41 \\
\hline $\mathrm{N} 2-\mathrm{H} 2 \cdots \mathrm{Ow} 4$ & 0.86 & 2.127 & $2.951(5)$ & 160.16 \\
\hline Ow1-HW1A $\cdots$ Ow $3^{\mathrm{ii}}$ & 0.90 & 2.050 & $2.802(4)$ & 140.43 \\
\hline 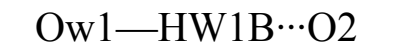 & 0.85 & 2.015 & $2.849(4)$ & 165.60 \\
\hline $\mathrm{Ow} 2-\mathrm{HW} 2 \mathrm{~A} \cdots \mathrm{O} 1$ & 0.82 & 2.058 & $2.826(4)$ & 155.82 \\
\hline Ow2-HW2B $\cdots \mathrm{O} 2^{\mathrm{iii}}$ & 0.90 & 2.222 & $3.000(6)$ & 144.96 \\
\hline Ow3-HW3A $\cdots$ Ow $1^{\text {iv }}$ & 0.84 & 2.196 & $2.742(4)$ & 122.43 \\
\hline $\mathrm{Ow} 3-\mathrm{HW} 3 \mathrm{~B} \cdots \mathrm{Ow} 2^{\mathrm{v}}$ & 0.83 & 2.282 & $3.107(6)$ & 172.46 \\
\hline Ow4-HW4A $\cdots \mathrm{N} 2$ & 0.86 & 2.207 & $2.951(5)$ & 144.03 \\
\hline Ow4-HW4B $\cdots$ Ow2 $2^{\mathrm{ii}}$ & 1.00 & 2.006 & $2.872(4)$ & 143.22 \\
\hline
\end{tabular}

Symmetry codes: (i) $\quad-x+1 / 2, y+1 / 2,-z+1 / 2$; (ii) $x+1 / 2,-y+1 / 2,-z+1 / 2$; (iii) I- $1 / 2,-y+1 / 2, z-1 / 2$; (iv) $-x-1 / 2, y-1 / 2,-z+1 / 2$; (v) $-x,-y,-z$; (vi) $-x-1 / 2, y-1 / 2,-z+1 / 2$. 
The water molecules play a crucial role for the stabilization of the crystal structure. They are responsible for the formation of an extensive three-dimensional hydrogen bonding network between themselves and the tetrahydroisoquinoline-1-one molecules (Table 1). This arrangement suggests similarity between the conformation in crystalline hydrate state and in water solution, which can correlate with an in vivo conformation of the molecule. The obtained structural data can provide insight for similar isoquinolinones, for which the structures in solution are not known.

To further study the structure of acid 1, $a b$ initio and DFT calculations were performed using the GAUSSIAN 03 suite of programs. ${ }^{11}$ The geometry of $\mathbf{1}$ was elucidated by calculations at $\mathrm{HF} / 6-31+\mathrm{G}^{* *}$ and B3LYP/6-31+G** levels. The B3LYP/6-31+G** optimized geometry of zwitterionic form of acid $\mathbf{1}$ is presented on Figure 3. Selected interatomic distances are collected in Table 2 (the X-Ray numbering scheme is used). The obtained $a b$ initio and DFT results for the geometry of zwitterionic form of $\mathbf{1}$ are in agreement with the X-ray data.

Table 2. Selected experimental and calculated distances $(\AA)$ and bond angles (degrees) of the zwitterionic form of $\mathbf{1}$ and ap-6a

\begin{tabular}{cccccc}
\hline & \multicolumn{3}{c}{$\mathbf{1}$} & \multicolumn{2}{c}{ ap-6a } \\
\cline { 2 - 6 } Parameter & X-ray & B3LYP & HF & B3LYP & HF \\
\cline { 2 - 6 } Distances & & & & 1.400 & 1.390 \\
C1-C2 & $1.391(4)$ & 1.403 & 1.393 & 1.394 & 1.385 \\
C2-C3 & $1.374(4)$ & 1.391 & 1.381 & 1.399 & 1.388 \\
C3-C4 & $1.376(5)$ & 1.402 & 1.392 & 1.397 & 1.387 \\
C4-C5 & $1.378(4)$ & 1.394 & 1.383 & 1.400 & 1.389 \\
C5-C6 & $1.390(4)$ & 1.403 & 1.393 & 1.399 & 1.385 \\
C1-C6 & $1.387(4)$ & 1.399 & 1.382 & 1.495 & 1.495 \\
C1-C7 & $1.482(4)$ & 1.485 & 1.486 & 1.379 & 1.362 \\
C7-N1 & $1.357(4)$ & 1.391 & 1.373 & 1.495 & 1.483 \\
C8-N1 & $1.497(3)$ & 1.483 & 1.472 & 1.514 & 1.559 \\
C8-C9 & $1.544(4)$ & 1.560 & 1.555 & 1.234 & 1.513 \\
C6-C9 & $1.507(4)$ & 1.497 & 1.501 & 1.468 & 1.462 \\
C7-O1 & $1.239(3)$ & 1.233 & 1.205 & 1.549 & 1.427 \\
N1-C15 & $1.467(3)$ & 1.465 & 1.458 & 1.548 & 1.538 \\
C8-C11 & $1.530(4)$ & 1.563 & 1.545 & & \\
C9-C24 & $1.552(4)$ & 1.601 & 1.571 & 1.232 & 1.206 \\
C24-O2 & $1.243(4)$ & 1.243 & 1.221 & & \\
C24-O3 & $1.252(4)$ & 1.263 & 1.238 & 34.16 & 32.44 \\
Torsion angles & & & & & 49.09 \\
C7-N1-C8-C9 & 28.18 & 30.60 & 30.19 & & 0.65 \\
N1-C8-C9-C6 & 44.59 & 41.17 & 42.80 & 2.42 & \\
C1-C7-N1-C8 & 1.72 & 5.39 & & & \\
\hline
\end{tabular}


In the course of the present investigation derivatives of acid $\mathbf{1}$ were synthesized and studied as well (Scheme 2). Acid chloride 4 was synthesized in a separate step and subsequently reacted with $\mathrm{MeOH}$ to the ester 5. Different reaction conditions were tested to increase the yield of acid chloride and thence - of 5 . The best achieved yield was 53\% after purification by column chromatography. Attempts to prepare the same compound by esterification with methanol/ $\mathrm{H}_{2} \mathrm{SO}_{4}$ were unsuccessful. When the reaction was carried out in methanol in the presence of thionyl chloride, only a small amount of the ester $5(5 \%)$ was isolated.

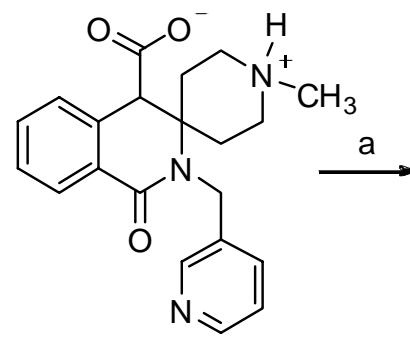

1

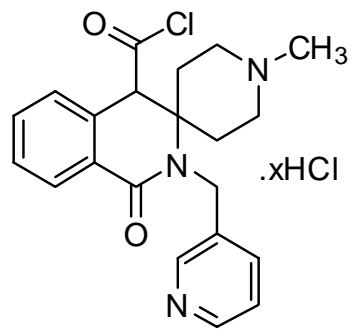

4<smiles>[R]C(=O)C1c2ccccc2C(=O)N(Cc2cccnc2)C12CCN(C)CC2</smiles>

5, 6a-e

\begin{tabular}{ccc}
\hline Entry & Secondary amine & Yield (\%) \\
\hline $6 \mathbf{6 a}$ & 42 \\
$\mathbf{6 b}$ & 46 \\
$\mathbf{6 c}$ & 53 \\
$\mathbf{6 d}$ &
\end{tabular}

Scheme 2. Reagents and conditions: (a) $\mathrm{SOCl}_{2}, \mathrm{DMF}, 75^{\circ} \mathrm{C}, \mathrm{C}_{6} \mathrm{H}_{6} / \mathrm{CHCl}_{3}$; (b) $\mathrm{CH}_{3} \mathrm{OH}, 60^{\circ} \mathrm{C}$; (c) Secondary amine, $\mathrm{THF}, 10^{\circ} \mathrm{C}$.

Amides 6a-e were also synthesized through $\mathbf{4}$ and various heterocyclic secondary amines ${ }^{4,12}$ (Scheme 2). It is worth noting that the obtained tetrahydroisoquinolines 6a-e contain four pharmacophoric groups: the lactam-amide fragment, pyridin-3-yl-methyl, N-methyl-piperidinyl and heterocyclic amido groups.

Structural studies on 1'-methyl-2-(pyridin-3-yl-methyl)-4-(pyrrolidine-1-carbonyl)-2,4dihydro-1H-spiro[isoquinoline-3,4'-piperidin]-1-one 6a were also performed. The geometries of the two possible isomers of $\mathbf{6 a}$ (with different orientation of pyrrolidine fragment) were located by calculations at $\mathrm{HF} / 6-31+\mathrm{G}^{* *}$ and $\mathrm{B} 3 \mathrm{LYP} / 6-31+\mathrm{G}^{* *}$ levels (Figure 2).

To estimate the solvent effect (water) on the relative stabilities of amides, the $\mathrm{PCM}^{13}$ method was applied, as implemented in the GAUSSIAN 03 suite of programs at $\mathrm{HF} / 6-31+\mathrm{G}^{* *}$ and B3LYP/6-31+G** levels for the geometry optimized at the same levels of theory (PCM/HF/6$31+\mathrm{G}^{* *} / / \mathrm{HF} / 6-31+\mathrm{G}^{* *}$ and PCM/B3LYP/6-31+G**//B3LYP/6-31+G** respectively). 

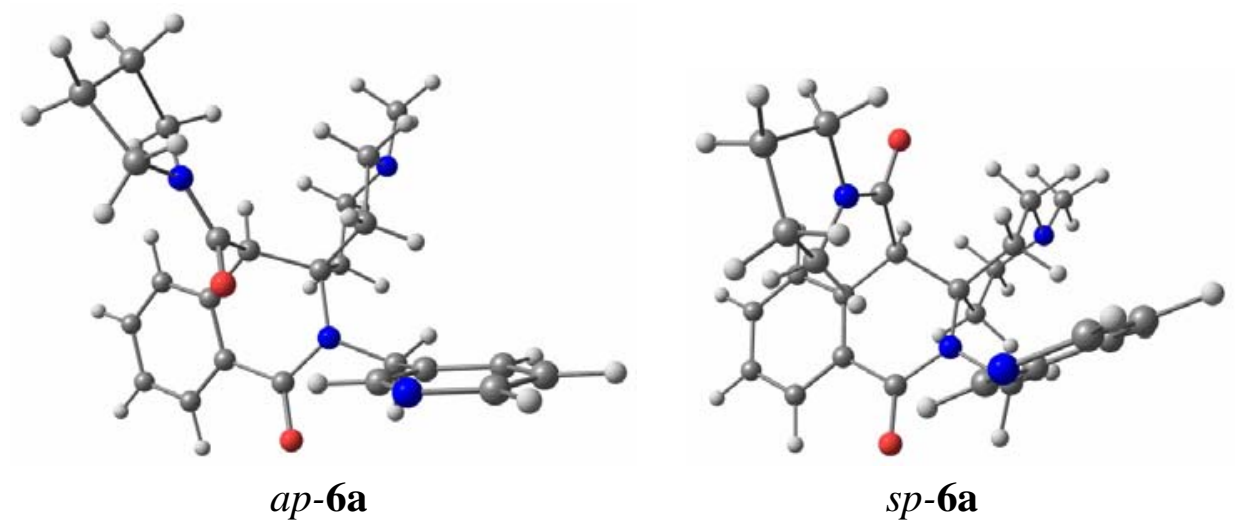

Figure 2. B3LYP optimized structures of amide 6a (antiperiplanar-(ap)- and synperiplanar-(sp)conformations of the proton $\mathrm{H}-4$ and amide carbonyl group).

Conformation ap-6a is preferred both in gas phase and in water solution (Table 3). The predicted geometry of ap-6a is very close to the geometry of unsubstituted compound $\mathbf{1}$ (see Figure 3 and Table 2).

Table 3. Relative energies $\Delta \mathrm{E}_{\mathrm{T}}\left(\mathrm{kcal} \mathrm{mol}^{-1}\right)$ of the isomers of amide 6

\begin{tabular}{ccccc} 
& \multicolumn{2}{c}{$\mathrm{HF} / 6-31+\mathrm{G}^{* *}$} & \multicolumn{2}{c}{$\mathrm{B} 3 \mathrm{LYP} / 6-31+\mathrm{G}^{* *}$} \\
\hline Species & Gas phase & $\mathrm{PC}$ & Gas phase & $\mathrm{PCM}$ \\
& & $\mathrm{M}$ & & \\
ap-6a & 0.00 & 0.00 & 0.00 & 0.00 \\
sp-6a & 1.70 & 5.29 & 0.71 & 4.11 \\
\hline
\end{tabular}
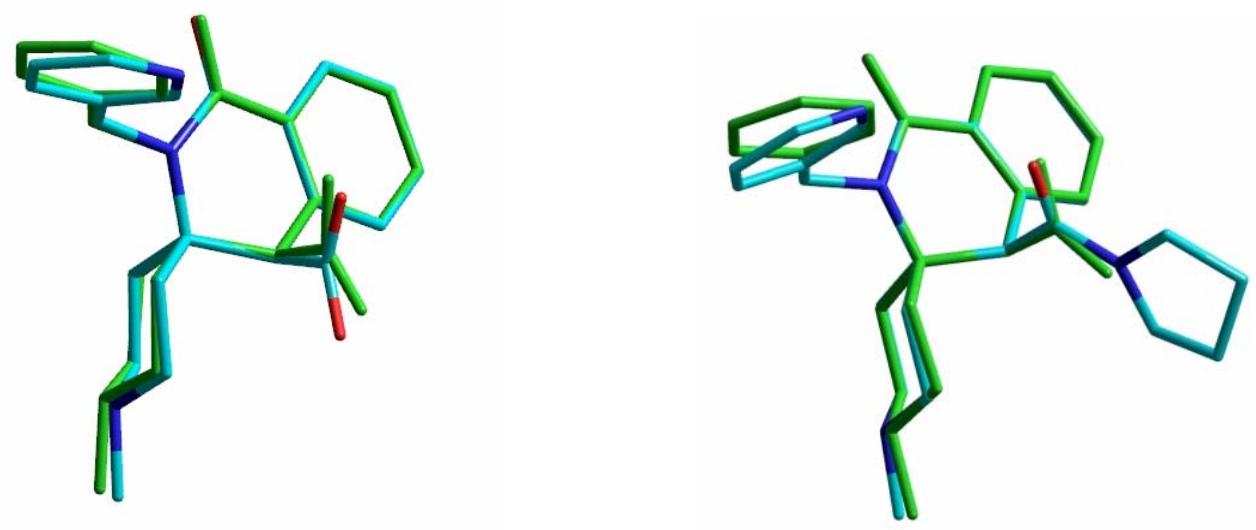

Figure 3. (A) Comparison of the X-ray determined structure of zwitterionic form of acid 1 (green) and the optimized one. (B) Comparison of the X-ray determined structure of 1 (green) and optimized structure of amide ap-6a. 
The $a b$ initio and DFT calculated bond distances and bond angles for zwitterionic form of 1'methyl-1-oxo-2-(pyridine-3-yl-methyl)-2,4-dihydro-1H-spiro[isoquinoline-3,4'-piperidine]-4carboxylic acid are in good agreement with the experimental ones. The calculations at the same levels performed for the 1'-methyl-2-(pyridin-3-yl-methyl)-4-(pyrrolidine-1-carbonyl)-2,4dihydro-1H-spiro[isoquinoline-3,4'-piperidin]-1-one suggest that the antiperiplanar conformer is favoured.

\section{Experimental Section}

General. All new synthesized compounds were purified by column chromatography and characterized on the basis of NMR and microanalytical data. NMR spectra were recorded on DRX Bruker Avance-250 $\left({ }^{1} \mathrm{H}\right.$ at $250.1 \mathrm{MHz} ;{ }^{13} \mathrm{C}$ at $\left.62.9 \mathrm{MHz}\right)$ and Bruker Avance II+ $600\left({ }^{1} \mathrm{H}\right.$ at $600.1 \mathrm{MHz} ;{ }^{13} \mathrm{C}$ at $150.9 \mathrm{MHz}$ ) spectrometer, with TMS as the internal standard; $J$ values are given in Hertz. Flash chromatography was performed on Silica Gel 60 (0.040-0.063 nm, Merck). Elemental analyses were performed by the Microanalytical Service Laboratory of Faculty of Chemistry, University of Sofia, using Vario EL3 CHNS(O). Melting points were determined on a Kofler hot stage.

For description of the NMR spectral data the arbitrary numbering given in formula of amide $\mathbf{6 c}$ is used (Figure 4).

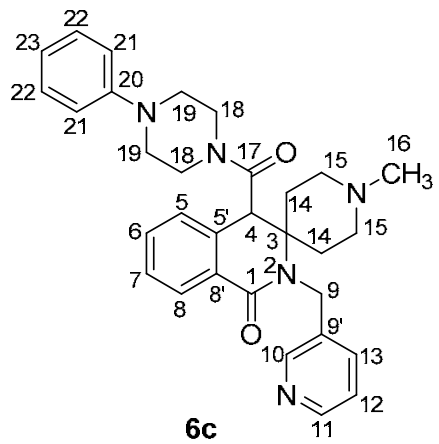

\section{Figure 4}

$N$-(1-Methylpiperidin-4-ylidene)-1-(pyridin-3-yl)methanamine (3). A mixture of equimolar amounts of 1-methylpiperidin-4-one and pyridin-3-ylmethanamine was heated at $100^{\circ} \mathrm{C}$ for 45 min. The pure product was isolated by vacuum distillation. Yield $76 \%$; bp $143-146^{\circ} \mathrm{C} / 2 \mathrm{mmHg}$; ${ }^{1} \mathrm{H}$ NMR $\left(250 \mathrm{MHz}, \mathrm{CDCl}_{3}\right): \delta 2.34\left(\mathrm{~s}, 3 \mathrm{H},-\mathrm{CH}_{3}\right), 2.27-2.32\left(\mathrm{~m}, 2 \mathrm{H},-\mathrm{CH}_{2}\right), 2.44-2.48(\mathrm{~m}, 2 \mathrm{H}$, $\left.\mathrm{CH}_{2}\right), 2.60-2.69\left(\mathrm{~m}, 4 \mathrm{H},-\mathrm{N}-\mathrm{CH}_{2}\right), 4.52\left(\mathrm{~s}, 2 \mathrm{H},=\mathrm{N}-\mathrm{CH}_{2}\right), 7.23-7.25$ (m, 1H, Py), 7.62-7.65 (m, $1 \mathrm{H}, \mathrm{Py}), 8.51-8.55(\mathrm{~m}, 2 \mathrm{H}, \mathrm{Py}) ;{ }^{13} \mathrm{C} \mathrm{NMR}\left(60 \mathrm{MHz}, \mathrm{CDCl}_{3}\right): \delta \quad 39.0\left(2 \mathrm{CH}_{2}\right), 45.7\left(\mathrm{CH}_{3}\right)$, 51.7(2 $\left.\mathrm{CH}_{2}\right), 55.4\left(=\mathrm{N}-\mathrm{CH}_{2}\right), 123.4,134.8,135.5,148.2(2 \mathrm{C}), 159.8(-\mathrm{N}=\mathrm{C}-)$.

1'-Methyl-1-oxo-2-(pyridin-3-yl-methyl)-2,4-dihydro-1H-spiro[isoquinoline-3,4'-piperidine]-4carboxylic acid (1). To a stirred suspension of homophthalic anhydride $2(0.0183 \mathrm{~mol})$ in $15 \mathrm{ml}$ 
dry $\mathrm{ClCH}_{2} \mathrm{CH}_{2} \mathrm{Cl}$ at room temperature the solution of heterocyclic ketimine $3(0.0183 \mathrm{~mol})$ in 10 $\mathrm{ml}$ dry $\mathrm{ClCH}_{2} \mathrm{CH}_{2} \mathrm{Cl}$ was added dropwise, and the mixture was stirred for $3 \mathrm{~h}$. The obtained solid was filtered and washed with $\mathrm{CH}_{2} \mathrm{Cl}_{2}$. Yield $93 \%$; mp 208-210 ${ }^{\circ} \mathrm{C}\left(\mathrm{CH}_{3} \mathrm{OH}\right)$; IR (nujol): 1660 (CON), 1610 ( $\left.\mathrm{COO}^{-} \mathrm{as}\right), 1410$ ( $\left.\mathrm{COO}^{-} \mathrm{s}\right) ;{ }^{1} \mathrm{H} \mathrm{NMR}$ (250 MHz, $\left.\mathrm{CF}_{3} \mathrm{COOD}\right): \delta 2.05-2.11$ (m, 2H, $14-\mathrm{H}), 2.33-2.43(\mathrm{~m}, 1 \mathrm{H}, 14-\mathrm{H}), 2.70-2.80(\mathrm{~m}, 1 \mathrm{H}, 14-\mathrm{H}), 3.00(\mathrm{~s}, 3 \mathrm{H}, 16-\mathrm{H}), 3.16-3.26(\mathrm{~m}, 1 \mathrm{H}$, 15-H), 3.41-3.51 (m, 1H, 15-H), 3.51-3.69 (m, 2H, 15-H), 4.56 (s, 1H, 4-H), 4.66 (d, 1H, 9-H, J $=17.2 \mathrm{~Hz}), 5.65(\mathrm{~d}, 1 \mathrm{H}, 9-\mathrm{H}, J=17.2 \mathrm{~Hz}), 7.38-7.40(\mathrm{~m}, 1 \mathrm{H}, 6-\mathrm{H}), 7.61-7.72(\mathrm{~m}, 2 \mathrm{H}, 5-, 7-\mathrm{H})$, 7.98-8.08 (m, 2H, 8-, 13-H), 8.57-8.68 (m, 2H, 11-, 12-H), $8.98(\mathrm{~s}, 1 \mathrm{H}, 10-\mathrm{H}) ;{ }^{13} \mathrm{C}$ NMR $(60$ $\left.\mathrm{MHz}, \mathrm{CF}_{3} \mathrm{COOD}\right): \delta 29.6$ (C-14), 30.2 (C-14), 42.8 (C-9), 43.7 (C-16), 47.5 (C-4), 51.3 (C-15), 51.5 (C-15), 58.4 (C-3), 126.2, 127.7, 128.7, 129.6, 130.8 (2C), 135.5, 139.5, 140.2 (C-12), 140.7 (C-10), 146.4 (C-11), 168.5 (C-1), 173.5 (C-17). Anal. Calcd for $\mathrm{C}_{21} \mathrm{H}_{23} \mathrm{~N}_{3} \mathrm{O}_{3}$ : C 65.78, $\mathrm{H}$ 6.57. Found: C 65.39, H 6.57.

Methyl 1'-methyl-1-oxo-2-(pyridin-3-yl-methyl)-2,4-dihydro-1H-spiro[isoquinoline-3,4'-piperidine]-4carboxylate (5). To a stirred suspension of acid $1(0.002 \mathrm{~mol})$ in $\mathrm{C}_{6} \mathrm{H}_{6} / \mathrm{CHCl}_{3}(1: 1)$ DMF was added $(0.0002 \mathrm{~mol})$. Then $\mathrm{SOCl}_{2}$ was added dropwise and the mixture was heated at $75^{\circ} \mathrm{C}$ for 2.5 h. The solvents were evaporated and to the residue of acid chloride $4 \mathrm{CH}_{3} \mathrm{OH}$ was added. The mixture was heated at $60^{\circ} \mathrm{C}$ for $1.5 \mathrm{~h}$. Then was cooled and the solvent was evaporated. The residue was dissolved in $10 \%$ aq. $\mathrm{Na}_{2} \mathrm{CO}_{3}$ and extracted with EtOAc, dried over anhydrous $\mathrm{MgSO}_{4}$. Evaporation of the solvent gave crude product. It was purified by flash-column chromatography (EtOAc: $\left.\mathrm{MeOH}: \mathrm{NH}_{3} / 4.7: 0.3: 0.08\right)$. Yield $53 \%$; mp 148-150 ${ }^{\circ} \mathrm{C}(\mathrm{EtOAc}) ;{ }^{1} \mathrm{H}-$ NMR $\left(600 \mathrm{MHz}, \mathrm{CDCl}_{3}\right): \delta 1.48(\mathrm{~d}, 1 \mathrm{H}, 14-\mathrm{H}, J=11.6 \mathrm{~Hz}), 1.66(\mathrm{dd}, 1 \mathrm{H}, 14-\mathrm{H}, J=13.8$, $2.5 \mathrm{~Hz}), 1.92(\mathrm{dt}, 1 \mathrm{H}, 14-\mathrm{H}, J=13.1,4.0 \mathrm{~Hz}), 2.11-2.15(\mathrm{~m}, 1 \mathrm{H}, 14-\mathrm{H}), 2.25-2.30$ (m, 1H, 15-H), $2.31(\mathrm{~s}, 3 \mathrm{H}, 16-\mathrm{H}), 2.39$ (t, 1H, 15-H , J = $11.9 \mathrm{~Hz}), 2.70(\mathrm{~d}, 1 \mathrm{H}, 15-\mathrm{H}, J=12.1 \mathrm{~Hz}), 2.76$ (d, $1 \mathrm{H}, 15-\mathrm{H}, J=11.9 \mathrm{~Hz}), 3.52(\mathrm{~s}, 3 \mathrm{H}, 18-\mathrm{H}), 4.34(\mathrm{~s}, 1 \mathrm{H}, 4-\mathrm{H}), 4.38$ (d, 1H, 9-H, J = $16.3 \mathrm{~Hz})$, 5.48 (d, 1H, 9-H, J=16.3 Hz), 7.22-7.27 (m, 2H, 5-H, 12-H), 7.46-7.51 (m, 2H, 6-H, 7-H), 7.49 $(\mathrm{dd}, 2 \mathrm{H}, J=5.8,9.0 \mathrm{~Hz}), 7.76(\mathrm{~d}, 1 \mathrm{H}, 13-\mathrm{H}, J=7.86 \mathrm{~Hz}), 8.19(\mathrm{dd}, 1 \mathrm{H}, 8-\mathrm{H}, J=7.2,1.5 \mathrm{~Hz})$, 8.49 (dd, 1H, 11-H, $J=4.5,1.02 \mathrm{~Hz}), 8.58(\mathrm{~d}, 1 \mathrm{H}, 10-\mathrm{H}, J=1.4 \mathrm{~Hz}) ;{ }^{13} \mathrm{C}-\mathrm{NMR}(150.9 \mathrm{MHz}$, $\mathrm{CDCl}_{3}$ ): $\delta 32.5(\mathrm{C}-14), 33.5$ (C-14), 42.0 (C-9), 45.8 (C-4) 48.3 (C-16), 51.5 (C-15), 52.0 (C-15), 52.4 (C-18), 59.4 (C-3), 123.3, 127.7, 128.7, 129.1, 132.4, 132.6, 135.1(2C), 135.7, 148.3, 165.4 (C-1), 170.3 (C-17). Anal. Calcd. for $\mathrm{C}_{22} \mathrm{H}_{25} \mathrm{~N}_{3} \mathrm{O}_{3}$ : C, 69.64; H 6.64. Found: C, 69.54; H 6.66.

\section{General procedure for the preparation of amides 6a-e}

To the solution of acid chloride $4(0.0013 \mathrm{~mol})$ in dry THF and dry $\mathrm{CHCl}_{3}(1: 1) 4.5$ eq. of the corresponding secondary amine were added at $10^{\circ} \mathrm{C}$ and the mixture was stirred at this temperature for $1 \mathrm{~h}$. Then was poured into $\mathrm{CH}_{2} \mathrm{Cl}_{2}(70 \mathrm{ml})$, washed with brine and dried over $\mathrm{MgSO}_{4}$. After evaporation of the solvent the crude product was purified by flash chromatography.

1'-Methyl-2-(pyridin-3-yl-methyl)-4-(pyrrolidine-1-carbonyl)-2,4-dihydro-1H-spiro[isoquinoline-3,4'piperidin]-1-one (6a). Yield $42 \%$ (after column chromatography); mp $144-146{ }^{\circ} \mathrm{C}$ (EtOAc); ${ }^{1} \mathrm{H}-$ NMR $\left(250 \mathrm{MHz}, \mathrm{CDCl}_{3}\right): \delta$ 1.60-1.86 (m, 5H, 14-H, 15-H, 19-H), 1.94-2.17 (m, 5H, 14-H, $15-$ 
H, 19-H), 2.23 (s, 3H, 16-H), 2.66-2.81 (m, 2H, 15-H), 3.33 (dt, 2H, 18-H, J = 7.0, 2.2 Hz), 3.743.87 (m, 2H, 18-H), 4.38 (s, 1H, 3-H), 4.67 (d,1H, 9-H, $J=16.6 \mathrm{~Hz}), 5.11(\mathrm{~d}, 1 \mathrm{H}, 9-\mathrm{H}, J=16.6$ $\mathrm{Hz})$, 7.15-7.26 (m, 2H, 5-H, 12-H), 7.33-7.44 (m, 2H, 6-H, 7-H), 7.95 (dm, 1H, 8-H, J = 11.98 $\mathrm{Hz}), 8.12-8.15(\mathrm{~m}, 1 \mathrm{H}, 13-\mathrm{H}), 8.40(\mathrm{dd}, 1 \mathrm{H}, 11-\mathrm{H}, J=4.7,1.4 \mathrm{~Hz}), 8.60(\mathrm{~d}, 1 \mathrm{H}, 10-\mathrm{H}, J=1.7$ $\mathrm{Hz}) ;{ }^{13} \mathrm{C}-\mathrm{NMR}\left(60 \mathrm{MHz}, \mathrm{CDCl}_{3}\right): \delta 24.2$ (C-19), 26.7 (C-19), 32.8 (C-14), $33.4(\mathrm{C}-14), 43.3(\mathrm{C}-$ 9), 45.4 (C-4), 46.3 (C-16), 46.5 (C-18), 47.9 (C-18), 52.4 (C-15), 52.6 (C-15), 59.8 (C-3), 123,7 (C-12), 127.0 (C-5), 128.5 (C-7), 129.6 (C-8), 129.8 (C-9'), 132.2 (C-6), 134.6, 135.6, 136.2 (C13), 148.1 (C-11), 148.6 (C-10), 165.6 (C-1), 168.3 (C-17). Anal. Calcd for $\mathrm{C}_{25} \mathrm{H}_{30} \mathrm{~N}_{4} \mathrm{O}_{2}$ : C 71.74; H 7.22. Found: C 71.54; H 7.58.

\section{1'-Methyl-4-(piperidine-1-carbonyl)-2-(pyridin-3-yl-methyl)-2,4-dihydro-1H-spiro[isoquinoline-}

3,4'-piperidin]-1-one (6b). Yield 46 \% (after column chromatography); mp 189-190 ${ }^{\circ} \mathrm{C}$ (EtOAc); ${ }^{1} \mathrm{H}$ NMR $\left(250 \mathrm{MHz}, \mathrm{CDCl}_{3}\right): \delta$ 1.65-1.85 (m, 6H, 19-H), 1.80-2.19 (m, 6H, 14-, 15-H), $2.27(\mathrm{~s}$, $3 \mathrm{H}, 16-\mathrm{H}), 2.66-2.78(\mathrm{~m}, 2 \mathrm{H}, 15-\mathrm{H}), 3.33-3.88(\mathrm{~m}, 4 \mathrm{H}, 18-\mathrm{H}), 4.66(\mathrm{~d}, 1 \mathrm{H}, 9-\mathrm{H}, J=16.5 \mathrm{~Hz})$, 4.68 (s, 1H, 4-H), 5.19 (d, 1H, 9-H, $J=16.5$ Hz), 7.11-7.16 (m, 1H, 5-H), 7.19 7.23 (m, 1H, 12$\mathrm{H})$, 7.39-7.46 (m, 2H, 6-, 7-H), 7.99-8.02 (m, 1H, 13-H), 8.17-8.21 (m, 1H, 8-H), 8.45 (dd, 1H, $11-\mathrm{H}, J=4.8,1.2 \mathrm{~Hz}), 8.61(\mathrm{~d}, 1 \mathrm{H}, 10-\mathrm{H}, J=1.7 \mathrm{~Hz}) ;{ }^{13} \mathrm{C} \mathrm{NMR}\left(60 \mathrm{MHz}, \mathrm{CDCl}_{3}\right): \delta 24.4(\mathrm{C}-$ 19), 25.4 (C-19), 27.1 (C-19), 32.1 (C-14), 33.0 (C-14), 41.4 (C-4), 43.0 (C-9), 43.3 (C-18), 45.6 (C-16), 47.9 (C-18), 51.9 (C-15), 52.2 (C-15), 59.3 (C-3), 123,4 (C-12), 126.8 (C-5), 128.1 (C7), 129.3 (C-8), 129.6 (C-9'), 131.9(C-6), 134.4, 135.2, 136.0 (C-13), 147.9 (C-11), 148,3 (C-10), 165.4 (C-1), 167.9 (C-17). Anal. Calcd for $\mathrm{C}_{26} \mathrm{H}_{32} \mathrm{~N}_{4} \mathrm{O}_{2}$ : C, 72.19; H, 7.46. Found: C, 71.83; H, 7.23 .

1'-Methyl-4-(4-phenylpiperazine-1-carbonyl)-2-(pyridin-3-yl-methyl)-2,4-dihydro-1H-

spiro[isoquinoline-3,4'-piperidin]-1-one (6c). Yield $55 \%$ (after column chromatography); mp 140-142 ${ }^{\circ} \mathrm{C}$ (EtOAc); ${ }^{1} \mathrm{H}-\mathrm{NMR}\left(600 \mathrm{MHz}, \mathrm{CDCl}_{3}\right): \delta$ 1.76-1.80 (m, 2H,14-H), 1.94-1.97 (m, 1H, $14 \mathrm{H}), 2.17-2.21(\mathrm{~m}, 1 \mathrm{H}, 15-\mathrm{H}), 2.25-2.30(\mathrm{~m}, 2 \mathrm{H}, 14-, 15-\mathrm{H}), 2.34(\mathrm{~s}, 3 \mathrm{H}, 16-\mathrm{H}), 2.76(\mathrm{~d}, 1 \mathrm{H}$, $15-\mathrm{H}, J=10.4 \mathrm{~Hz}), 2.85(\mathrm{~d}, 1 \mathrm{H}, 15-\mathrm{H}, J=10.9 \mathrm{~Hz}), 3.06-3.40(\mathrm{~m}, 4 \mathrm{H}, 18-\mathrm{H}), 3.66-3.76(\mathrm{~m}, 2 \mathrm{H}$, 19-H), 4.03-4.09 (m, 2H, 19-H), $4.71(\mathrm{~s}, 1 \mathrm{H}, 3-\mathrm{H}), 4.71(\mathrm{~d}, 1 \mathrm{H}, 9-\mathrm{H}, J=16.1 \mathrm{~Hz}) 5.21$ (d, 1H, $J=$ 16.1 Hz), 6.91-6.96 (m, 3H, 22-, 23-H), 7.19 (d, 1H, 5-H, J=6.96 Hz), 7.27-7.32 (m, 3H, 12-, 21-H), 7.44-7.49 (m, 2H, 6-, 7-H), 8.00 (d, 1H, 13-H, J=7.6 Hz), 8.22 (d, 1H, 8-H J = 7.0 Hz), 8.49 (bs, 1H, 11-H), 8.67 (bs, 1H, 10-H); ${ }^{13} \mathrm{C}-\mathrm{NMR}\left(150.9 \mathrm{MHz}, \mathrm{CDCl}_{3}\right): \delta 32.6(\mathrm{C}-14), 33.4$ (C-14), 41,8 (C-4), 42.4 (C-19), 43.4 (C-9), 46.1 (C-16), 47.3 (C-19), 49.5 (C-18), 50.8 (C-18), 52.4 (C-15), 52.6 (C-18), 59.7 (C-3), 116.7 (2C-22), 117.0, 120.9 (C-23), 123,6 (C-12), 126.8 (C-5), 128.4 (C-7), 129.3 (C-5), 129.5 (C-8), 129.6 (C-9'), 132.2 (C-6), 134.0 (C-8'), 136.1 (C13), 148.0 (C-11), 148.4 (C-10), 150.6 (C-20), 165.4 (C-1), 168.2 (C-17). Anal. Calcd for $\mathrm{C}_{31} \mathrm{H}_{35} \mathrm{~N}_{5} \mathrm{O}_{2}$ : C, 73.06; H, 6.92. Found: C, 72.80; H, 7.03.

4-(4-(4-Fluorophenyl)piperazine-1-carbonyl)-1'-methyl-2-(pyridin-3-yl-methyl)-2,4-dihydro-1Hspiro[isoquinoline-3,4'-piperidin]-1-one (6d). Yield 53 \% (after column chromatography); mp 134$136{ }^{\circ} \mathrm{C}$ (EtOAc); ${ }^{1} \mathrm{H}-\mathrm{NMR}\left(600 \mathrm{MHz}, \mathrm{CDCl}_{3}\right): \delta 1.71-1.76(\mathrm{~m}, 2 \mathrm{H}, 14-\mathrm{H}), 1.90(\mathrm{td}, 1 \mathrm{H}, 14-\mathrm{H}, \mathrm{J}=$ $13.5,4.2 \mathrm{~Hz}), 2.03-2.07(\mathrm{~m}, 1 \mathrm{H}, 15-\mathrm{H}), 2.10-2.14(\mathrm{~m}, 1 \mathrm{H}, 14-\mathrm{H}), 2.22(\mathrm{td}, 1.0 \mathrm{H}, 15-\mathrm{H}, J=12.8$, $4.2 \mathrm{~Hz}), 2.28(\mathrm{~s}, 3 \mathrm{H}, 16-\mathrm{H}), 2.70-2.71(\mathrm{~d}, 1 \mathrm{H}, 15-\mathrm{H}, J=11.6 \mathrm{~Hz}), 2.79$ (d, 1H, 15-H, $J=12.2$ 
$\mathrm{Hz}), 2.92-3.0(\mathrm{~m}, 2 \mathrm{H}, 18-\mathrm{H}) 3.15-3.20(\mathrm{~m}, 2 \mathrm{H}, 18-\mathrm{H}), 3.61-3.67$ (m, 2H, 19-H), 3.98-4.06 (m, 2H, 19-H), $4.65(\mathrm{~d}, 1 \mathrm{H}, 9-\mathrm{H}, J=16.5 \mathrm{~Hz}), 4.67(\mathrm{~s}, 1 \mathrm{H}, 3-\mathrm{H}), 5.22$ (d, 1H, 9-H, J = $16.5 \mathrm{~Hz})$, 7.13-7.16 (m, 1H), 7.24-7.26 (m, 1H), 7.41-7.4 (m, 2H, 6-, 7-H), 7.99 (d, 1H, 13-H, J = 7.9 Hz), 8.22 (d, 1H, 8-H J=7.0 Hz), 6.87-6.89 (m, 2H, 22-H), 6.97-7.01 (m, 2H, 5-, 12-H, J=6.96 Hz), 7.27-7.32 (m, 3H, 12-, 21-H), 7.44-7.49 (m, 2H, 6-, 7-H), 8.00 (d, 1H, 13-H, J = 7.6 Hz), 8.22 (dd, 1H, 8-H $J=7.2,1.7 \mathrm{~Hz}), 8.47(\mathrm{~d}, 1 \mathrm{H}, 11-\mathrm{H}, J=3.7 \mathrm{~Hz}), 8.63(\mathrm{~d}, 1 \mathrm{H}, 10-\mathrm{H}, J=1.5 \mathrm{~Hz})$; ${ }^{13} \mathrm{C}-\mathrm{NMR}\left(150.9 \mathrm{MHz}, \mathrm{CDCl}_{3}\right): \delta 32.6$ (C-14), 33.3 (C-14), 41,2 (C-4), 42.1 (C-19), 42.9 (C-9), 46.1 (C-16), 47.0 (C-19), 50.2 (C-18), 51.5 (C-18), 52.2 (C-15), 52.5 (C-18), 59.5 (C-3), 115.7 (C-22), 115.9 (C-22), 118.6, 118.7, 123.5 (C-12), 126.8 (C-5), 128.4 (C-7), 129.6 (C-8), 129.7 (C-9'), 132.1 (C-6), 134.0 (C-8'), 135.2, 136.1 (C-13), 147.2 (C-20), 148.1 (C-11), 148.4 (C-10), 157.0, 158.6, 165.2 (C-1), 168.2 (C-17). Anal. Calcd for $\mathrm{C}_{31} \mathrm{H}_{34} \mathrm{FN}_{5} \mathrm{O}_{2}$ : C, 70.57; H, 6.50.; Found: C, 70.73; H, 6.64.

1'-Methyl-4-(morpholine-4-carbonyl)-2-(pyridin-3-yl-methyl)-2,4-dihydro-1H-spiro[isoquinoline3,4'-piperidin]-1-one (6e). Yield $65 \%$ (after column chromatography); mp 180-182 ${ }^{\circ} \mathrm{C}$ (EtOAc); ${ }^{1} \mathrm{H}$ NMR $\left(250 \mathrm{MHz}, \mathrm{CDCl}_{3}\right): \delta$ 1.69-2.30 (m, 6H, 14-, 15-H), 2.28 (s, 3H, 16-H), 2.69-2.84 (m, 2H, 15-H), 3.48-3.87 (m, 8H, 18-, 19-H), 4.60 (s, 1H, 4-H), 4.67 (d, 1H, 9-H, J = 16.5 Hz), 5.19 (d, 1H, 9-H, $J=16.5 \mathrm{~Hz}), 7.08-7.12(\mathrm{~m}, 1 \mathrm{H}, 6-\mathrm{H}), 7.25-7.29$ (m, 1H, 13-H), 7.42-7.48 (m, 2H, 5, 7-H), 7.98-8.01 (m, 1H, 8-H), 8.19-8.23 (m, 1H, 12-H), 8.47 (d, 1H, 11-H, J = 3.9Hz), 8.63 (s, $1 \mathrm{H}, 10-\mathrm{H}) ;{ }^{13} \mathrm{C}$ NMR (60 MHz, $\left.\mathrm{CDCl}_{3}\right): \delta 32.4$ (C-14), 33.1 (C-14), 41,0 (C-4), 42.5 (C-18), 42.9 (C-9), 45.9 (C-16), 47.4 (C-18), 49.5 (C-18), 52.0 (C-15), 52.3 (C-15), 59.4 (C-3), 66.8 (2C19), 123,4 (C-12), 126.8 (C-5'), 128.3 (C-7), 129.4 (C-8), 129.6 (C-9'), 132.0 (C-6), 133.9 (2C), 135.9 (C-13), 147.9 (C-11), 148.3 (C-10), 165.1 (C-1), 168.3 (C-17). Anal. Calcd for $\mathrm{C}_{25} \mathrm{H}_{30} \mathrm{~N}_{4} \mathrm{O}_{3}$ : C, 69.10; H, 6.96. Found: C, 68.97; H, 7.35.

\section{Acknowledgements}

Thanks are due to Johnson \& Johnson, R\&D, Janssen Pharmaceutica, Belgium, for financial support of the project and to Prof. Mariana Palamareva, Faculty of Chemistry, University of Sofia, for useful comments on the manuscript.

\section{References and Notes}

1. (a) Scott, J.; Williams, R. M. Chem. Rev. 2002, 102, 1669. (b) Lin, H.-Ru; Safo, M. K.; Abraham, D. J. Bioorg. Med. Chem. Lett. 2007, 17 (9), 2581. (c) Saito, N.; Koizumi, Y.; Tanaka, C.; Suwanborirux, K.; Amnuoypol, S.; Kubo A. Heterocycles 2003, 61, 79. (d) 
Rothweiler, U.; Czarna, A.; Krajewski, M.;Ciombor, J.; Kalinski, C.; Khazak, V.; Ross, G.; Skobeleva, N.; Weber, L.; Holak, T. A. Chem. Med. Chem. 2008, 3, 1118.

2. (a) Gitto, R.; Ferro, S.; Agnello, S.; De Luca, L.; De Sarro, G.; Russo, E.; Vullo, D.; Supuran, C. T.; Chimirri, A. Bioorg. Med. Chem. 2009, 17, 3659. (b) Gitto, R.; Barreca, M. L.; Francica, E.; Caruso, R.; De Luca, L.; Russo, E.; De Sarro, G.; Chimirri, A. Arkivoc 2004, 5, 170. (c) Yang, J.; Hua, W.-Y.; Wang, F.-X.; Wang, Z.-Y.; Wang, X. Bioorg. Med. Chem. 2004, 12 (24), 6547. (d) Okuda, K.; Kotake, Y.; Ohta, S. Bioorg. Med. Chem. Lett. 2003, 13 (17), 2853. (e) Yamada, N.; Kadowaki, S.; Takahashi, K.; Umeu, K. Biochem. Pharmacol. 1992, 44, 1211. (f) Houlihan, W. J.; Gogerty, J. H.; Parrino, V. A.; Ryan, E. J. Med. Chem. 1983, 26, 765.

3. (a) Kametani, T. The Chemistry of the Isoquinoline Alkaloids, Elsevier: New York, 1969. (b) Xu, Xi-Y.; Qin, G.-W.; Xu, R.-S.; Zhu, X.-Z. Tetrahedron 1998, 54, 14179. (c) Fontana, A.; Cavaliere, P.; Wahidulla, S.; Naik, C. G.; Cimino G. Tetrahedron 2000, 56, 7305.

4. Stoyanova, M. P.; Angelova, S. E.; Kosev, K. S.; Denkova, P. S.; Enchev V. G.; Palamareva, M. D. Tetrahedron Lett. 2006, 47, 2119.

5. (a) Dalence-Guzmarn, M. F; Berglund, M.; Skogvall, S.; Sterner, O. Bioorg. Med. Chem. 2008, 16, 2499. (b) Lane, J. W.; Estevez, A.; Mortara, K.; Callan, O.; Spencerc, J. R.; Williams R. M. Bioorg. Med. Chem. Lett. 2006, 16, 3180. (c) Aubry, S.; Pellet-Rostaing, S.; Fenet, R.; Lemaire, M. Tetrahedron Lett. 2006, 47, 1319.

6. (a) Haimova, M. A.; Mollov, N. M.; Ivanova, S. C.; Dimitrova, A. I.; Ognyanov, V. I. Tetrahedron 1977, 33, 331. (b) Georgieva, A.; Stanoeva, E.; Karamfilova, K.; Spassov, S.; Angelova, O.; Haimova, M.; Kimpe, N. De; Boelens, M. Tetrahedron 1994, 50, 9399. (c) Bonnaud, B.; Carlessi, A.; Bigg, D. C. H. J. Heterocycl. Chem. 1993, 30, 257. (d) Yadav, J.; Reddy, B.; SarithaRaj, K.; Prasad, A. Tetrahedron 2003, 59, 1805. (e) Azizian, J.; Mohammadi, A. A.; Karimi, A. R.; Mohammadizadeh, M. R.; Koohshari, M. Heterocycles 2004, 63, 2013. (f) Gonzalez-Lopez, M.; Shaw, J. T. Chem. Rev. 2009, 109, 164.

7. (a) Kozekov, I. D.; Koleva, R. I.; Palamareva, M. D. J. Heterocycl. Chem. 2002, 39, 229. (b) Stoyanova, M. P.; Kozekov, I. D.; Palamareva, M. D. J. Heterocycl. Chem. 2003, 40, 795. (c) Kandinska, M. I.; Kozekov, I. D.; Palamareva, M. D. Molecules 2006, 11, 403. (d) Burdzhiev, N.; Stanoeva, E. Tetrahedron 2006, 62, 8318. (e) Burdzhiev, N.T.; Stanoeva, E. R. Z. Naturforsch. B: Chem. Sci. 2008, 63, 313. (f) Christov, P. P.; Kozekov I. D.; Palamareva, M. D. J. Heterocycl. Chem. 2006, 43, 1015. (g) Bogdanov, M. G.; Gocheva, B. T.; Dimitrova, D. B.; Palamareva, M. D. J. Heterocycl. Chem. 2007, 44, 673.

8. Cushman, M.; Gentry, J.; Dekow, F. J. Org. Chem. 1977, 42, 1111.

9. The supplementary crystallographic data for 1 (CCDC 743286) can be obtained free of charge via www.ccdc.cam.ac.uk/conts/retrieving.html (or from the Cambridge Crystallographic Data Centre, 12 Union Road, Cambridge CB2 1EZ, UK; fax: (+44) 1223336-033; or deposit@ccdc.cam.ac.uk).

10. (a) Petrova, R.; Shivachev, B.; Kosev, K.; Stoyanova, M.; Angelova, S. Acta Cryst. 2005, E61, o2248. (b) Kandinska, M. I.; Todorov, I. S.; Shivachev, B.; Bogdanov, M. G. Acta 
Cryst. 2007, E63, o2544. (c) Nikolova, R. P.; Kolev, T.; Statkova-Abeghe, S. M.; Shivachev, B. L. Acta Cryst. 2008, E64, o1796. (d) Yildirim, S. Ö.; Akkurt, M.; Kandinska, M. I.; Bogdanov, M. G.; Büyükgüngör, O. Acta Cryst. 2008, E64, o1932.

11. Frisch, M. J.; Trucks, G. W.; Schlegel, H. B.; Scuseria, G. E.; Robb, M. A.; Cheeseman, J. R.; Montgomery, J. A., Jr.; Vreven, T.; Kudin, K. N.; Burant, J. C.; Millam, J. M.; Iyengar, S. S.; Tomasi, J.; Barone, V.; Mennucci, B.; Cossi, M.; Scalmani, G.; Rega, N.; Petersson, G. A.; Nakatsuji, H.; Hada, M.; Ehara, M.; Toyota, K.; Fukuda, R.; Hasegawa, J.; Ishida, M.; Nakajima, T.; Honda, Y.; Kitao, O.; Nakai, H.; Klene, M.; Li, X.; Knox, J. E.; Hratchian, H. P.; Cross, J. B.; Adamo, C.; Jaramillo, J.; Gomperts, R.; Stratmann, R. E.; Yazyev, O.; Austin, A. J.; Cammi, R.; Pomelli, C.; Ochterski, J. W.; Ayala, P. Y.; Morokuma, K.; Voth, G. A.; Salvador, P.; Dannenberg, J. J.; Zakrzewski, V. G.; Dapprich, S.; Daniels, A. D.; Strain, M. C.; Farkas, O.; Malick, D. K.; Rabuck, A. D.; Raghavachari, K.; Foresman, J. B.; Ortiz, J. V.; Cui, Q.; Baboul, A. G.; Clifford, S.; Cioslowski, J.; Stefanov, B. B.; Liu, G.; Liashenko, A.; Piskorz, P.; Komaromi, I.; Martin, R. L.; Fox, D. J.; Keith, T.; Al-Laham, M. A.; Peng, C. Y.; Nanayakkara, A.; Challacombe, M.; Gill, P. M. W.; Johnson, B.; Chen, W.; Wong, M. W.; Gonzalez, C.; Pople, J. A. Gaussian 03, Revision B.04, Gaussian, Inc., Pittsburgh PA 2003.

12. Haimova, M.; Atanasova, I.; Stanoeva, E.; Mihovska, S. Comm. Dept. Chem. Bulg. Acad. Sci. 1984, 17, 163.

13. (a) Cancès, M. T.; Mennucci, B.; Tomasi, J.; J. Chem. Phys. 1997, 107, 3032. (b) Mennucci, B.; Tomasi J. J. Chem. Phys. 1997, 106, 5151. 